Questions vives

\section{Questions Vives}

Recherches en éducation

Vol. $8 n^{\circ} 15$ | 2011

Égalité filles-garçons : où en est-on?

\title{
La vigilance des enseignant-e-s d'éducation physique et sportive relative à l'égalité des filles et des garçons
}

Physical education teachers' vigilance towards gender equity

\section{Catherine Patinet-Bienaimé et Geneviève Cogerino}

\section{OpenEdition}

\section{Journals}

\section{Édition électronique}

URL : http://journals.openedition.org/questionsvives/765

DOI : $10.4000 /$ questionsvives.765

ISBN : 978-2-8218-1090-7

ISSN : $1775-433 \mathrm{X}$

\section{Éditeur}

Université Aix-Marseille (AMU)

\section{Édition imprimée}

Date de publication : 15 septembre 2011

ISBN : 978-2-912643-39-1

ISSN : 1635-4079

Référence électronique

Catherine Patinet-Bienaimé et Geneviève Cogerino, « La vigilance des enseignant-e-s d'éducation physique et sportive relative à l'égalité des filles et des garçons », Questions Vives [En ligne], Vol.8 n $^{\circ}$ 15 | 2011, mis en ligne le 10 octobre 2011, consulté le 01 mai 2019. URL : http:// journals.openedition.org/questionsvives/765; DOI : 10.4000/questionsvives.765

Ce document a été généré automatiquement le 1 mai 2019.

\section{(†) $\odot$

Questions Vives est mis à disposition selon les termes de la licence Creative Commons Attribution Pas d'Utilisation Commerciale - Pas de Modification 4.0 International. 


\title{
La vigilance des enseignant-e-s d'éducation physique et sportive relative à l'égalité des filles et des garçons
}

\author{
Physical education teachers' vigilance towards gender equity
}

Catherine Patinet-Bienaimé et Geneviève Cogerino

\section{Le cours d'EPS, lieu de renforcement des inégalités de sexe}

1 La mise en place tardive de la mixité des cours en EPS n'a pas été accompagnée d'une réflexion concernant l'éducation à l'égalité des sexes. Les prescriptions rappelées dans la charte interministérielle de 2000 et 2006 sont peu connues des enseignant-e-s d'EPS et ne font pas l'objet de façon systématique d'un plan de formation initiale ou continue les concrétisant. De plus, l'intégration des activités physiques et sportives (APS) dès la fin des années 60 comme support de la discipline a concouru à privilégier une «culture sportive » structurée autour d'un modèle de "puissance et de performance » (Coakley, 2004), à valence masculine. Encore aujourd'hui les enseignant-e-s d'EPS choisissent un éventail restreint d'APS valorisant un modèle sportif compétitif peu attractif pour de nombreuses filles (Combaz \& Hoiban, 2008). Tout ceci ne fait que renforcer les différences, les élèves ayant eu d'inégales probabilités d'accès à la pratique sportive en dehors de l'école selon leur origine sociale et en particulier le diplôme de leurs parents (Louveau, 2002). L'évaluation lors des épreuves du baccalauréat a été et reste révélatrice d'inégalités persistantes (Cleuziou, 2000 ; Vigneron, 2006) en faveur des garçons.

Dans le même temps de nombreux travaux ethnographiques ou études du discours liés aux gender-studies confirment des perceptions et prises de décision des enseignant-e-s s'appuyant majoritairement sur une idéologie essentialiste et androcentrique. Les filles 
dans le contexte masculin de l'EPS sont, pour les enseignant-e-s, "des problèmes", manquant de motivation, ne faisant aucun effort et étant uniquement préoccupées par leur apparence. Le discours fait apparaître nettement leur « manque » en rapport avec ce que sont les garçons : manque de courage, manque de compétence, manque de qualités physiques. Les enseignant-e-s sont également à l'origine de plaisanteries vis-à-vis des capacités ou morphologies de leurs élèves ou créent des sous-entendus renforçant les stéréotypes (Rǿnholt, 2002). Ces constats (stéréotypisation sexuée, dichotomisation et naturalisation des compétences féminines ou masculines) ont été établis dans de nombreux pays, que l'EPS y soit enseignée en contexte mixte ou unisexe (Berg \& Lalema, 2010 ; Davis, 2003 ; Garett, 2004 ; Koca, 2009 ; Larsson, Fagreel \& Redelius, 2009 ; Beyria \& Guérandel, 2010; Wright, 1996). Tous ces auteurs mettent en cause l'origine sportive de l'éducation physique et son appui sur les APS comme facteur principal.

3 En EPS, Cogérino et Lechelard (2003) se sont attachées à caractériser les dimensions qui déterminent l'attribution de l'attention de l'enseignant-e d'EPS à certains élèves plus qu'à d'autres. Il ressort que les enseignant-e-s sont sensibles à certains critères, conformes aux stéréotypes sexués. Selon qu'ils sont garçons ou filles, les élèves ne sont pas remarqués pour les mêmes caractéristiques. Les filles sont davantage citées pour les caractéristiques négatives de leurs prestations ou ressources et pour les caractéristiques positives de leurs comportements. Les garçons sont remarqués pour les caractéristiques positives de leurs prestations et les caractéristiques négatives de leurs comportements. Cette vision stéréotypée qu'ont les enseignant-e-s d'EPS des élèves est confirmée par les résultats de l'enquête réalisée par la DEPP ${ }^{1}$ en 2005-2006 (Benhaïm-Grosse, 2007) : les problématiques des capacités physiques et de la motivation sont clairement posées pour justifier les différences de réussite.

4 Couchot-Schiex et Trottin (2005) démontrent que le nombre d'interactions lors des séances d'EPS est bien en faveur des garçons. Si les filles et les garçons reçoivent autant de feed-back permettant la transmission des informations, en revanche les garçons reçoivent davantage de consignes leur permettant de structurer la situation d'apprentissage, d'organiser leur travail.

5 Bonniot-Paquien, Cogérino et Champely (2009) ont étudié les élèves présentant des comportements hors tâche ou passifs en EPS. Les résultats montrent que huit comportements de garçons sur dix entraînent une intervention de l'enseignant-e contre seulement la moitié des comportements identiques de filles. Les garçons ne présentent pas de façon significative plus de comportements hors tâche que de comportements passifs. Pourtant, les enseignant-e-s interrogé-e-s décrivent les garçons comme agités et les filles comme passives, attestant d'attitudes professionnelles marquées par les stéréotypes sexués.

6 Enfin, plusieurs enquêtes qualitatives (Beyria \& Guérandel, 2010; Vigneron, 2004) attestent que les comportements des garçons sont l'étalon à partir duquel les enseignante-s gèrent les séances. Les formes de mixité sont induites par ce que l'on peut appeler « la loi des garçons » qu'il s'agit de ne pas frustrer.

7 Les stéréotypes de sexe ne s'expriment pas qu'au travers d'activités masculines. Coltice (2005) montre qu'une situation dansée, même si elle offre au départ des ouvertures vers des positionnements masculins et féminins n'implique pas de façon magique ces expériences variées. Cette conformité aux stéréotypes de sexe est également constatée dans les cours de cirque de lycée dans une "cité » de la périphérie urbaine de Lyon (Garcia, 2007). Les pratiques sont décrites comme très sexuées en liaison avec les modèles 
dominants de la masculinité et de la féminité (la prouesse pour les garçons ; la souplesse et la composition chorégraphique pour les filles). La hiérarchie, le respect de l'ordre des genres (Connell, 1987) sont bien réels tant d'un point de vue physique que symbolique. Ainsi les garçons accaparent le matériel permettant les prouesses d'équilibre. Leurs performances physiques lors de leur passage "sur scène » sont valorisées, alors que les filles tiennent souvent des rôles de faire-valoir des exploits de leurs camarades garçons. Les enseignant-e-s d'EPS interrogé(e)s justifient leurs choix par l'«égalité dans la différence » entre filles et garçons et la « complémentarité des sexes » qui favoriseraient l'activité des élèves.

8 L'ensemble de ces travaux démontrent comment professeurs et élèves contribuent à l'intériorisation ou l'émancipation de rôles sociaux conventionnellement attribués à leur sexe respectif et définissent ainsi un contexte d'enseignement favorisant ou non l'égalité des sexes (Lee, Marks \& Byrd, 1994).

\section{Des expériences de mixité différentes en EPS}

On ne peut parler d'égalité des sexes sans évoquer la spécificité des contextes de mixité vécus par les enseignant-e-s et les élèves en EPS. La dimension corporelle, occultée quand les élèves sont assis derrière une table de classe, est évidente en EPS. Les corps sont bien présents même si le corps désirant y est, comme dans les autres disciplines, largement dénié (Pujade-Renaud, 1983). Pour les élèves, la mixité constitue une expérience subjective particulière. Exposés au regard des autres élèves, ils prennent conscience d'appartenir à un groupe de sexe dominant ou dominé. Pour certaines jeunes filles la motivation à pratiquer rejoint des raisons essentiellement utilitaristes : avoir un corps féminin, sexuellement attirant, correspondant aux normes idéales proposées par la société médiatique (Garett, 2004). Se sentir trop grosse ou avoir peur de sentir mauvais peuvent alors être autant de raisons d'éviter la pratique. La peur de s'afficher en public en EPS ne fait que renforcer cette pression de l'idéal corporel ainsi que l'image donnée de capacités et performances moindres. Ces jeunes filles ressentent ainsi un véritable " déficit de féminité » (Cockburn \& Clarke, 2002) et craignent d'être jugées (Garett, 2004). Du côté des garçons, la pression pour toujours être à la hauteur est forte (Davison, 2000) : si un garçon peut scolairement avoir des difficultés, sur le terrain de sport, il est en compétition permanente avec les autres pour montrer sa virilité. Certains en souffrent, d'autres le font au détriment des plus faibles.

Du côté des enseignant-e-s, la mixité a une image globalement positive (Artus, 1999): travailler en mixité permettrait d'accepter l'altérité, diminuer les préjugés, prendre en compte toutes les différences, s'affirmer, se maîtriser, avoir de la curiosité d'esprit. Terret, Cogérino, et Rogowski (2006) estiment que les enseignant-e-s se positionnent sur une mixité plus pédagogique et éthique que didactique. Majoritairement, la mixité est perçue comme favorisant le climat de classe. L'« apprendre à vivre ensemble » est associé à la finalité de construction d'une vraie citoyenneté. Le "caractère d'évidence » de la mixité (Artus, 1999) est remis en question dans la réalisation des groupes au sein de la classe (ce qui est très fréquent dans cette discipline) : quand faut-il ou non démixer? Différentes modalités sont proposées: la séparation des deux sexes, les groupes de niveaux (les garçons sont le plus souvent dans les groupes forts), les groupes de besoins (réunis autour de niveaux d'habiletés proches), les groupes affinitaires, les groupes hétérogènes avec ou sans aménagement. En définitive, si la mixité du cours d'EPS n'est 
plus remise en question (comme c'était encore le cas dans les années 80/90), à l'intérieur du cours, l'enseignant-e opte pour une gestion de mixité qui lui est propre. Sa perception subjective du contexte amène à des intentions différentes de mixité qui influencent sa vigilance relative à l'égalité des sexes.

11 A la lecture de ces constats, on pourrait croire que les enseignant-e-s désireux-ses majoritairement de faire réussir les filles et les garçons n'ont pas conscience des discriminations qu'ils contribuent à mettre en place. C'est ce caractère d'évidence d'actes non conscients que nous avons cherché à questionner au travers de l'attention déployée en cours par les enseignant-e-s d'EPS. De quoi les enseignant-e-s ont-ils/elles conscience quand ils/elles enseignent en mixité ? A quoi font-ils/elles attention? Quelle vigilance relative à l'égalité des sexes exercent-ils/elles?

\section{Attention et Conscience en acte}

12 Nous utilisons le terme "conscience» dans sa dimension phénoménologique, expérientielle et non dans sa dimension morale, de jugement sur nous-mêmes. La langue anglaise distingue deux termes "awareness" et "consciousness» quand le français ne donne que le mot «conscience». Depraz (2002) rappelle que le terme awareness signifie une "conscience de base, minimale, graduelle et ouverte, présence à soi non-inconsciente» (Depraz, 2002, p. 12). Alors que consciousness est une conscience plus réflexive. Le terme conscience renvoie donc à ces deux orientations, une attention au sens de vigilance ouverte et une activité réflexive. Husserl (1950) distingue la conscience réfléchie de la conscience pré-réfléchie, ou conscience directe. La conscience pré-réfléchie n'est pas inconscience mais non conscience de «je » en même temps qu'elle est conscience du monde. Elle est donc antérieure à toute mise en mots et permet à l'individu de fonctionner de façon automatique. La prise de conscience est alors le passage entre l'activité pré-réfléchie à l'activité réfléchie, permettant la verbalisation.

Cet éclairage théorique permet d'approcher des niveaux de conscience différents des enseignant-e-s d'EPS. S'ils (elles) n'ont pas une conscience réfléchie des discriminations qu'ils (elles) contribuent à mettre en place, notre hypothèse est qu'ils/elles ne sont pas sans conscience pré-réfléchie des éléments liés à ces discriminations et que nous pouvons documenter ce premier niveau en faisant expliciter l'attention déployée lors de moments de cours mixtes.

14 L'attention, dans son approche subjective est conçue comme «ce qui module la conscience de » (Vermersch, 2002). En choisissant le point de vue de l'attention, on peut décrire les propriétés fonctionnelles de la conscience, sa structure. L'attention est un objet dynamique qui apparaît par ses changements contrastés (Vermersch, 2002). Deux fonctions de l'attention cohabitent entre ce qui est visé (le " prendre pour thème ») et ce qui est remarqué ("le remarqué »). La visée principale de l'attention peut être ainsi capturée par des saillances exogènes des contenus propres à chaque type d'acte (par exemple, une fille inactive alors que l'enseignant-e est centré-e sur la conduite du cours) ou des prégnances endogènes provenant des significations sédimentées du fait des expériences déjà vécues (par exemple, une aversion pour les filles non sportives). Ainsi, à tout moment, des éléments périphériques peuvent passer au centre et le thème attentionnel initial passer à la périphérie. 


\section{Méthodologie}

15 L'échantillon a été constitué de 24 enseignant-e-s de collège de façon à équilibrer $1 /$ le nombre d'hommes et de femmes, 2/le nombre d'années d'ancienneté dans l'enseignement, 3/ le type d'établissement («normal », REP, Zone sensible, rural), 4/ le niveau de diplôme, les recherches réalisées montrant une différenciation des représentations de la mixité selon les sexes, générations, diplômes (Artus, 1999 ; Terret et al., 2006).

Des entretiens de «ressouvenir» d'expériences passées (Vermersch, 1994), ont été conduits avec la technique de «l'entretien d'explicitation» (EdE). La question initiale de mise en évocation était la suivante: " je te propose, si tu en es d'accord, de retrouver un moment de cours dans lequel tu t'es senti-e [tu ne t'es pas senti-e] capable de faire apprendre ensemble les filles et les garçons ».

Il est indispensable que les données se rapportent à un vécu de référence (ce qui s'est passé « ce jour-là, à ce moment-là ») pour éviter tout discours sur des généralités. La technique de recueil de données exige que l'interviewé rende à nouveau présent à sa conscience son vécu. Tout le long de l'EdE le chercheur doit garder cette qualité d'évocation qui est la condition d'un accès à un vécu passé. Enfin, l'EdE repose sur un questionnement non inductif où le chercheur veille à reprendre les mots, les verbes, le vocabulaire de l'enseignant-e en cherchant à élucider le déroulement de l'action, en encourageant la description et en bannissant la question du « pourquoi ? ». Les relances se situent: 1 / sur un axe chronologique pour décrire le début, le moment, la fin, avec des relances comme: "Comment tu as commencé? Et ensuite?»; 2/ en cherchant une granularité de la description. Par exemple: «Et quand tu fais... qu'est ce que tu fais?»; $3 /$ soit en cherchant à identifier les prises d'informations, les décisions, les effectuations. Par exemple : «Et quand...à quoi tu sais que? Et là, à quoi es-tu attentif?»; 4/ soit en cherchant les différentes structures feuilletées de l'attention. Par exemple : «Et tout en ... qu'est ce que tu fais aussi? ".

Les critères de mise en évocation ont été prioritaires: tout entretien (ou partie d'entretien) portant sur des généralités a été éliminé. Les matériaux recueillis comportent des indices perceptifs permettant de savoir "qui » les enseignant-e-s percevaient (classe, groupe, élèves, garçons, filles), et quels thèmes attentionnels ils/elles poursuivaient. Ces indices perceptifs sont nettement différenciés: pour les garçons, vitesse excessive, tensions, cris, bousculades, détournement de la situation, insultes, regards pour défier l'enseignant-e ; pour les filles, attitude à l'arrêt, manque de mobilité, placement dans un espace périphérique, recul devant les situations d'opposition, peur des engins etc.

Du point de vue du traitement des données, six catégories descriptives ont été retenues : 1 / les thèmes attentionnels; $2 /$ les actes qui précisent le thème attentionnel ; $3 /$ les contenus attentionnels; 4 / les croyances et préjugés sexistes; $5 /$ les émotions; $6 /$ les arrière-plans à la situation.

La description de chaque moment vécu permet d'identifier à quoi les enseignant-e-s d'EPS font attention quand ils/elles font cours en mixité. L'interprétation des thèmes attentionnels (intention d'égalité ou d'inégalité) est réalisée grâce à la catégorisation de 
formes typiques d'intervention privilégiant l'égalité/ l'inégalité des sexes (cf. tableau $\mathrm{n}$ $\left.{ }^{\circ} 1\right)$.

Tableau 1 : Catégorisation de formes typiques d'intervention privilégiant l'égalité/ l'inégalité des sexes (d'après Lee, Marks \& Byrd, 1994)

\begin{tabular}{|c|c|}
\hline Vers l'égalité & Vers l'inégalité \\
\hline $\begin{array}{l}\text { - absence de préjugés envers les filles et } \\
\text { les garçons; } \\
\text { - répartition des rôles et vigilance par } \\
\text { rapport à la domination des garçons; } \\
\text { - équilibre en quantité et en qualité des } \\
\text { interventions en faveur des deux sexes; } \\
\text { - mise en place et la régulation de } \\
\text { situations équitables; } \\
\text { - valorisation de toutes les réussites, quel } \\
\text { que soit le sexe; } \\
\text { - intervention sur toute attitude/ } \\
\text { remarque/insulte sexiste ou homophobe; } \\
\text { - installation de la mise en confiance dans } \\
\text { les situations de mixité. }\end{array}$ & $\begin{array}{l}\text { - affichage des préjugés envers les filles et les } \\
\text { garçons; } \\
\text { - mise en place et/ ou non-régulation de situations } \\
\text { favorisant la domination des garçons; } \\
\text { - déséquilibre en quantité et en qualité de } \\
\text { l'intervention en faveur d'un seul sexe ; } \\
\text { - recours systématique à la discrimination } \\
\text { positive; } \\
\text { - stigmatisation d'un(e) élève ou un groupe d'élève } \\
\text { par rapport à son sexe; } \\
\text { - non-intervention face à une attitude ou une } \\
\text { insulte sexiste; } \\
\text { - renforcement d'un climat de classe peu favorable } \\
\text { à la confiance mutuelle entre garçons et filles. }\end{array}$ \\
\hline
\end{tabular}

21 La présentation des résultats procède en deux temps. Le premier temps rassemble de manière synthétique les résultats du corpus. Le second temps cherche à illustrer les résultats au travers des extraits de deux études de cas.

\section{Une faible vigilance relative à l'égalité des sexes}

Les résultats montre une grande variabilité intra et interindividuelle envers l'égalité. Au cours d'une même expérience, l'attention des enseignant-e-s est en mouvement, passe d'un type à l'autre, est soumise aux aléas de la situation, ses propriétés dynamiques, son imprévisibilité. Même si une intention de départ oriente l'attention vers l'égalité, de nombreux remarqués secondaires émergent et réorientent le cours de l'attention. Tout au long d'un cours, les enseignant-e-s passent d'une absence totale de vigilance relative à l'égalité à des éveils attentionnels envers elle. Ceci ne signifie pas qu'ils ne sont pas sans conscience directe de ce qui se joue du point de vue de l'égalité. Ils perçoivent (dans la conscience directe) des éléments porteurs d'égalité ou de discrimination, mais ces éléments ne sont pas systématiquement recherchés dans le cours d'action.

Si certain(e)s enseignant-e-s ont décrit une attention soutenue pour rechercher l'égalité ou prévenir les risques de discrimination, le plus souvent, nous ne pouvons pas parler de vigilance. Quand l'attention est orientée vers l'égalité, c'est avec un caractère fragile et contingent.

Les contenus attentionnels qui parviennent à l'enseignant-e sont toujours interprétés au filtre de sa subjectivité, son expérience passée, et ses stéréotypes. Les enseignant-e-s sont sensibles à l'écart à la norme sportive en fonction du sexe des élèves. Etre une fille pourrait rendre plus apparent et insupportable l'écart à cette norme très prégnante en EPS. De nombreux enseignant-e-s d'EPS sont en attente d'indicateurs masculins : la force, 
la vitesse, l'habileté, l'intelligence sont autant de qualités recherchées chez les garçons et décrites comme absentes chez les filles. La perception des filles est donc réalisée «en creux » par rapport à cette référence masculine. Le discours est émaillé de préjugés sexistes envers les filles plus importants qu'envers les garçons. L'acceptation des différences et le rapport à l'altérité est également variable. Pour certaines enseignantes, le réveil d'expériences passées négatives avec des filles peu actives (y compris quand elles étaient élèves) conduit à une posture de rejet d'autant plus forte que ces filles ne sont pas à leur image. Ces enseignantes "sportives", présentant des attentes et des comportements proches de ceux de leurs collègues sportifs masculins, ne se reconnaissent guère dans l'inertie, le rejet des contenus d'enseignement ou de la discipline EPS qui caractérisent certaines filles. Enfin, les émotions liées au sentiment d'incompétence face à certaines situations de mixité semblent orienter l'enseignant-e vers un recours aux préjugés sexistes pour « sortir la tête haute ».

\section{Un lien étroit entre type de mixité et égalité des sexes}

La perception subjective du contexte oriente l'enseignant-e vers des intentions différentes de mixité propre à l'expérience vécue. Chaque enseignant-e pratique ainsi une forme de mixité singulière en lien avec chaque contexte rencontré. Quatre formes typiques ont été identifiées : une mixité « ensemble-séparée »; une mixité "banalisée »; une mixité "recherchée »; une mixité "réfléchie». Chacune d'entre elle porte des logiques intrinsèques et favorise des formes d'égalité des sexes différentes.

Dans la mixité "ensemble-séparée », la séparation en deux espaces ségrégués des garçons et des filles est initiée par l'enseignant-e ou par les élèves eux-mêmes de façon tacite. C'est donc une mixité en apparence (Goffman, 1977; Faure \& Garcia, 2003). L'enseignant-e pratique une double attention entre un « ici » et un «là-bas » peu propice à la vigilance, et déséquilibrant les interventions au profit d'un des deux sexes. Le type d'égalité développée fait nettement appel à un modèle d'égalité différencialiste et la référence au masculin, omniprésente, n'avantage pas les filles. Les enseignant-e-s sont moins ambitieux-ses envers elles, persuadé(e)s qu'elles n'ont pas les moyens pour réussir.

Dans la mixité banalisée, les élèves cohabitent sans aménagement particulier. La mixité banalisée repose sur la croyance en une équivalence ou une complémentarité des sexes. Les enseignant-e-s sont dans l'illusion d'une " égalité de traitement ». Dans ce contexte "d'équivalence » favorisant le masculin, les garçons retrouvent leur attitude dominatrice (réduisant l'activité des filles) sans que les enseignant-e-s n'interviennent.

Dans la mixité recherchée, l'enjeu pour l'enseignant-e est que les filles et les garçons pratiquent ensemble. Les formes d'égalité présentes correspondent souvent, lors des jeux d'équipe avec ballon, à des aménagements de règles ${ }^{2}$ qui avantagent les filles (« égalité compensatoire »). L'attention principale est centrée sur l'instauration de la mixité et occulte la recherche de critères permettant d'identifier et différencier les comportements des garçons et des filles.

Enfin, on trouve certaines mixités réfléchies pour lesquelles les conditions de la mixité sont alors davantage questionnées. L'enseignant-e peut procéder à une recherche active d'indices, de signes précurseurs des discriminations. Ils/elles sont vigilant-e-s à la fois à contrôler les garçons dans leurs attitudes dominatrices et à aider les filles pour qu'elles s'engagent davantage. Même s'il/elle y a recours, il/elle doute de la fragilité des 
dispositifs de tutorat ou de discrimination positive. Le regard porté sur les filles en difficulté est davantage empathique que dans les autres types de mixité.

\section{Deux illustrations : Agnès et Michel}

Existe-t-il des situations subjectivement plus favorables que d'autre pour la vigilance relative à l'égalité sexuée? La comparaison de deux études de cas nous permet de montrer comment les facteurs subjectifs jouent en faveur du maintien ou de la distraction de la vigilance relative à l'égalité des sexes.

\section{Agnès : le risque de stigmatisation comme alerte de la vigilance}

31 Agnès explicite un moment de cours dont le support est un match de base-ball. La classe est répartie en deux équipes mixtes hétérogènes. L'histoire de la classe fait apparaître un aménagement de règle pour frapper la balle : il est permis de bénéficier de conseils d'un membre de son équipe lors des 3 essais autorisés. Dans une première étape du récit, la vigilance de l'enseignante concerne l'arbitrage de la partie de base-ball. Puis, Agnès perçoit que c'est Julie, en grande difficulté motrice, qui va devoir batter alors qu'il y a déjà eu deux élèves éliminés dans l'équipe. Elle entend les remarques de prophétie d'élimination de la jeune fille avant toute intervention de celle-ci. Elle ne les commente pas, restant dans un rôle d'arbitre-organisateur, cherchant volontairement à masquer ses émotions.

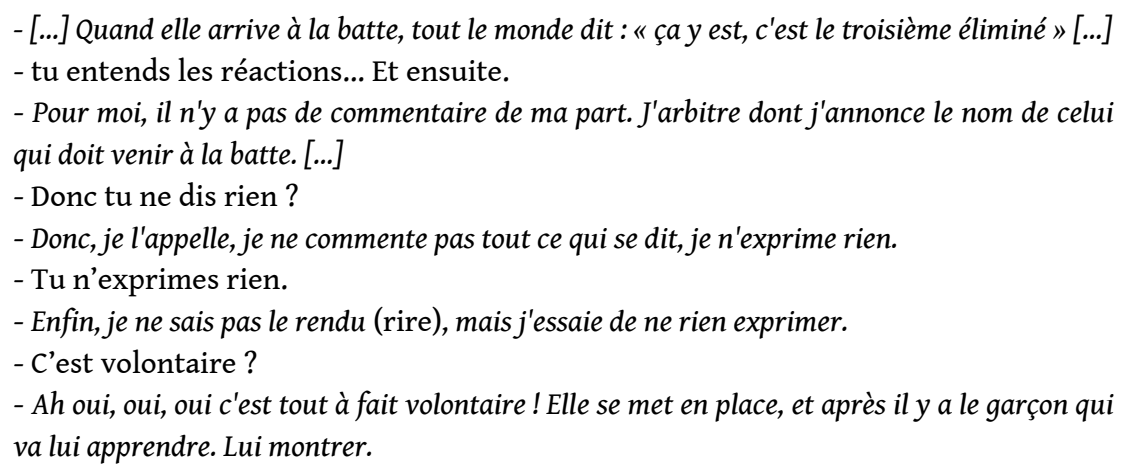

Agnès accepte l'aide proposée par un garçon de l'équipe. Elle contrôle l'interaction, en vérifie la précision, la justesse. Le maintien en prise sur une vigilance relative à l'égalité se poursuit par des actes d'autorisation à distance, de laisser-faire en donnant confiance, de signes fins pour approuver l'aide du garçon tuteur (la bonne distance, les bons appuis, les bons moments).

- [...] Et ensuite?

- Il descend, il va la placer.

- « Il va la placer », c'est-à-dire?

- C'est-à-dire, qu'il la met à bonne distance, il la manipule, hein. Il lui met la batte sur l'épaule, il lui dit: « tu regardes, tu te mets de profil », voilà.

- Tu fais attention à quoi, toi, à ce moment-là ?

- Je fais attention à ce que le lanceur ne lance pas. Je lui dis : « tu attends qu'elle soit prête »... et puis, j'encourage.

- Tu encourages?

- Je dis: « oui, c'est ça, oui, il a raison, oui c'est bien »... enfin, j'encourage, et puis j'attends (rire) [...]. 
Agnès développe une stratégie de guidage à distance. Son intervention discrète dévolue les savoirs aux élèves. La verbalisation subjective de l'expérience montre un déplacement de l'attention de la conduite du cours vers la recherche d'égalité. Agnès maintient une partie de son attention sur la dissimulation de ses émotions. Elle cherche à ne rien exprimer quand c'est Julie qui se présente pour batter, ni quand elle entend les remarques de sa probable élimination. Elle revient dans son rôle d'arbitre quand Julie rate son premier essai. Elle félicite Julie sans commenter l'exploit, percevant qu'elle est au bord des larmes.

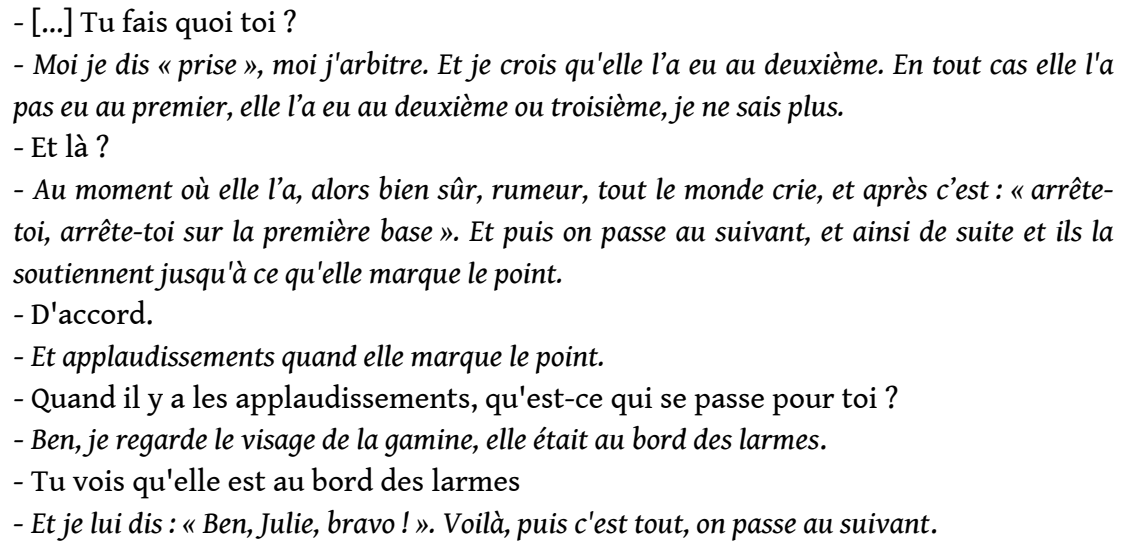

Cet extrait d'explicitation montre comment, chez certain-e-s enseignant-e-s, le risque de stigmatisation d'une élève fille agit comme une véritable alerte pour intervenir vers l'équité. Mais cet extrait est également exemplaire d'un contexte d'intervention mixte favorable à l'instauration de l'égalité : l'histoire de la classe, construite pour cette activité, autour de règles d'entraide, constitue pour l'enseignante un arrière-plan de mise en confiance dans les situations mixtes, ce qui favorise le maintien en prise vers des décisions d'égalité des sexes. Deux facteurs se combinent alors favorablement: la connaissance des risques discriminatoires et la mise en place d'un contexte de mixité favorable à une forme d'équité.

\section{Les préjugés sexistes et la mixité séparée}

Lors d'une séance de football avec une classe de $6^{\text {ème }}$ mixte, Michel souhaite mettre en place une situation qu'il a l'habitude de faire en club et installe son matériel tout en laissant les filles et les garçons s'échauffer.

- [...] Et toi tout en installant les plots, tu perçois ?...

- Oui, en périphérique, dans mon dos, je sens que les filles ne contrôlent pas le ballon et que

les garçons se font des grandes passes. Alors déjà j'interviens, je dis : « bon! Les garçons, c'est

des passes de 10 m maximum. [...]

- Et les filles?

- Et les filles, c'est : «j'ai du mal à me déplacer avec la balle et je m'applique », ça par contre, il y a de l'application de la part des filles.

Michel perçoit les comportements différents entre filles et garçons. Ces comportements opposés le font douter de la pertinence de la situation qu'il veut mettre en place suite à son échauffement.

- Et là, je me rends compte que la situation est difficile pour elles. Et je m'en rends compte. Et je suis en train de me dire : "non, tu ne devrais pas faire cela ", " tu devrais te concentrer làdessus». Donc, je suis en train de me dire ça et en même temps il y a les garçons qui 
balancent des grands ballons et je leur dis : "non pas des grands ballons ». Et, je m'énerve un peu. Et puis finalement je veux mettre en place ma situation [...]. la situation, d'un autre il sait que sa compréhension sera difficile. Il choisit alors huit garçons pour faire démontrer la situation.

- Et là, je me dis : " pour expliquer, il ne faut pas que ce soit que du verbal, sinon ils ne vont rien comprendre ». Donc, je prends huit élèves et je montre avec ces huit élèves.

- Tu prends qui ?

- Naturellement, pour être sûr, je prends que des garçons, (rire) conscience d'avoir agi sans équité et préfère pratiquer une légère autodérision pour garder la face. Nous estimons que le choix de huit garçons pour faire la démonstration est induit à la fois par les difficultés perçues à propos des filles mais surtout par les préjugés négatifs de Michel vis-à-vis de celles-ci. Ayant effectué ensuite deux groupes de sexes pour réaliser l'exercice, l'enseignant est partagé entre filles et garçons. Sa première urgence est de rendre autonome les garçons pour pouvoir consacrer ensuite son temps aux filles.

- Je lance ici, je vais là-bas, je fais des observations ici, et je reviens ici. Quand je siffle ça tourne, même si je suis là-bas. Je n'ai pas besoin d'être à côté. Donc je reste un petit moment pour leur expliquer cela. Ça va, ils ont compris. Et après, je vais voir les filles. Et en fait pendant que je fais ça, les filles commencent. Et en fait, je sens enfin je ne sais pas pourquoi mais j'ai un pressentiment, je sens qu'il y en a qui sont assises ou alors qui se questionnent en fait.

L'intention de l'enseignant est de faciliter une gestion qu'il pressent d'emblée difficile dans deux espaces séparés. Tout en agissant envers les garçons, les filles sont présentes dans une actualité potentielle. L'enseignant perçoit des filles assises ou qui se questionnent.

- Et, effectivement quand j'arrive je suis obligé de dire : « bon allez, il faut commencer, on y va» et là, il y en a une qui me dit: «mais je fais quoi, moi ?» [...] Donc, comme elle me demandait : «je fais quoi moi », en position d'appui, celle qui était au centre avait compris. Et les autres regardaient. Ça va, c'est joli (rire) et «nous on fait quoi?» [...]

Il va alors s'occuper d'elles et développe un thème attentionnel entièrement orienté vers leur aide, afin qu'elles comprennent la situation et son intérêt. Après plusieurs explications longues et inefficaces de la situation, Michel, se sentant en échec, réactive ses préjugés sexistes :

- Les garçons, c'est normal qu'ils aient compris tout de suite parce que c'est une activité qu'ils font des fois en dehors. Et ça leur plaît, voilà. Alors, tout de suite, ils percutent. Les filles, c'est plus difficile, parce que ce n'est pas une activité dans laquelle elles rentrent tout de suite. [...] ça ne leur correspond pas.

41 Si l'enseignant réalise une focalisation de son attention pour aider les filles, des remarqués concernant les garçons distraient son attention :

- et pendant ce temps les garçons en périphérique ça tourne mais ça foire aussi et j'entends :

"il y en a marre de ce jeu ».

Progressivement l'attention envers les filles va être détournée au profit des garçons avec des indices précis sur leur détournement de la situation.

Cet extrait d'explicitation montre comment, lors de moments de cours, de nombreuses préoccupations endogènes ou exogènes peuvent distraire certain-e-s enseignant-e-s de la vigilance relative à l'égalité des sexes: l'alerte concernant les difficultés des filles ne 
fonctionne pas. Cet extrait est également exemplaire d'une séparation en deux groupes de sexe dont la gestion est problématique. Enfin symboliquement, l'activité football active les préjugés positifs chez les garçons (« tout de suite, ils percutent ») et négatif chez les filles ("ça ne leur correspond pas »). Trois facteurs se combinent alors défavorablement : la nonprise en compte du niveau débutant dans l'activité football, les préjugés sexistes envers les filles et les garçons dans un contexte très marqué d'un point de vue masculin et enfin, la mise en place d'un contexte de mixité défavorable à la gestion équitable des deux sexes.

\section{Discussion} renforcer ses préjugés sexistes, soit lui permettre de lire finement l'interaction entre les filles et les garçons. Tout se passe de façon très schématique de la façon suivante : ce qui capte l'attention de l'enseignant-e, ce sont des signes proches des stéréotypes sexués. Pour Agnès, il s'agit d'une fille en échec qui risque d'être stigmatisée. Pour Michel, il s'agit des comportements fortement différenciés des filles et des garçons en football. Ensuite, la lecture de l'activité réelle des élèves, de leur interaction, du risque de discrimination peut réveiller une vigilance relative à l'équité. Alors que l'absence de cette lecture, pour différentes raisons, enferme dans les préjugés et les stéréotypes. Pour Agnès le risque de stigmatisation est bien perçu, le suivi attentionnel du tutorat à distance permet de déjouer ce risque. Pour Michel, la volonté de réaliser son plan de séance occulte une véritable anticipation des comportements des filles face à la situation proposée. Devant l'échec de la situation, les préjugés sexistes sont renforcés.

$$
\text { propositions pour que les enseignant-e-s s'exercent à être vigilant(e)s envers l'égalité }
$$
sexuée.

Tout d'abord, la vigilance nécessite la connaissance des risques discriminatoires propres aux situations d'enseignement mixte de l'EPS. Les enseignant-e-s doivent apprendre à identifier un certain nombre de scénarios pédagogiques favorisant ces risques. 
Ensuite, la formation devrait chercher à ce que les enseignant-e-s prennent conscience de leurs gestes professionnels équitables ou sexistes en lien avec le type de mixité pratiquée. L'analyse des pratiques professionnelles devrait questionner chacun sur ses propres préjugés sexistes et sur sa lecture de l'activité réelle des filles et des garçons.

Il faudrait également renforcer dans les publications didactiques la description différenciée de tous les profils d'élèves : garçons, filles, sportifs, non-sportifs afin d'aider les enseignant-e-s à passer de préjugés valorisant le masculin à une approche prenant en compte toutes les différences, y compris les différences liées au sexe. Un entraînement systématique à cette lecture différenciée est indispensable.

Enfin, les enseignant-e-s sont souvent en "panne» sur le «comment faire?». Des recherches devraient expérimenter et analyser des formes de pratiques scolaires mixtes plus égalitaires.

\section{BIBLIOGRAPHIE}

Artus, D. (1999). La mixité : illusion égalitaire en Education Physique et Sportive ? Son étude à travers les représentations des enseignants et des lycéens. Thèse de doctorat, STAPS, Université de Poitiers.

Benhaïm-Grosse, J. (2007). Image du sport scolaire et pratiques d'enseignement au lycée et au collège, 2005-2006. DEPP, Les dossiers, 190.

Berg, P., \& Lahelma, E. (2010). Gendering processes in the field of physical education, Gender and Education, 22(1), 31-46.

Beyria, F., \& Guerandel, C. (2010). La mixité dans les cours d'EPS d'un collège en ZEP : entre distance et rapprochement des sexes. Revue française de pédagogie, 170, 17-30.

Bonniot-Paquien, N., Cogérino, G., \& Champely, S. (2009). Les enseignants d'EPS face aux élèves qui décrochent de l'activité : interventions selon le sexe des élèves et discours relatifs aux comportements observés. STAPS, 84, 77-92.

Cleuziou, J.-P. (2000). L'analyse du menu et des notes. In B. David (Ed.), Education Physique et sportive : la certification au baccalauréat (pp. 77-124). Paris : INRP.

Coakley, J. (2004). Sport in society : Issues and Controversies (8ème edition), New York : McGrawhill.

Cockburn, C. \& Clarke, G. (2002). Everybody's looking at you ! Girls negotiating the "femininity deficit" they incur in physical education. Women's studies international forum, 25(6), 651-665.

Cogérino, G. (2005). (Ed). Filles et garçons en EPS. Paris : Revue EP.S.

Cogérino, G. (2006). La mixité en Education Physique et Sportive : de la gestion des groupes à une réflexion sur l'équité. In G. Cogérino (Ed.), La mixité en Education Physique, Paroles, réussites, différenciations (pp. 9-27). Paris : Revue EP.S.

Cogérino, G. \& Lechelard, F. (2003). Catégorisation sociale des élèves et stéréotypes de sexe en EP ? Communication aux journées d'étude de la Société Française de Psychologie du Sport, Paris, Charléty, mars 2003. 
Coltice, M. (2005). Danse et identité de genre. In G. Cogérino (Ed.), Filles et garçons en EPS (pp. 101-124). Paris : Editions Revue EP.S.

Combaz, G. \& Hoiban, O. (2008). Le rôle de l'école dans la construction des inégalités de sexe. Travail, genre et sociétés, 20, 129-150.

Connell, R. W. (1987). Gender and Power : Society, the Person and Sexual Politics. Cambridge : Polity Press.

Couchot-Schiex, S. \& Trottin, B. (2005). Interactions enseignants/élèves : variations en fonction du sexe et du genre. In G. Cogérino (Ed.), Filles et garçons en EPS (pp. 163-182). Paris : Revue EP.S.

Davis, K. (2003). Teaching for gender equity in physical education : a review of the literature. Women in sports \& physical activity journal, 12(2), 55-82.

Davison, K. G. (2000). Boys' Bodies in School : Physical Education. Journal of men's studies, 8(2), 255-263.

Depraz, N. (2002). La conscience. Approches croisées, des classiques aux sciences cognitives. Paris : Armand Colin/VUEF.

Evans, J., Davies, B., \& Penney, D. (1996). Teachers, teaching and the social construction of gender relations. Sport, education and society, 1(2), 165-183.

Faure, S., \& Garcia, M.-C. (2003). Les « braconnages » de la danse HIP HOP dans les collèges de quartiers populaires. Ville-Ecole-Intégration, 133, 244-257.

Garcia, M.-C. (2007). Représentations « genrées » et sexuation des pratiques circassiennes en milieu scolaire. Sociétés et Représentations, 24, 129-143.

Garrett, R. (2004). Negotiating a physical identity : girls, bodies and physical education, Sport, education and society, 9(2), 223-237.

Goffman, E. (1977). The arrangement between the sexes. Theory and Society, 4(3), 301-331.

Hauw, D. (2000). Les groupes en EPS. In J.-P. Rey (Ed.), Le groupe (pp. 89-104). Paris : Editions Revue EP.S.

Koca, C. (2009). Gender interactions in coed physical education : a study in Turkey, Adolescence, 44, 165-185.

Larsson, H., Fagrell, B., \& Redelius, K. (2009). Queering physical education. Between benevolence towards girls and tribute to masculinity. Physical education and sport pedagogy, 14(1), 1-17.

Lee, V. E., Marks, H. M., \& Byrd, T. (1994). Sexism in single-sex and coeducational independent secondary school classrooms. Sociology of education, 67(2), 92-120.

Louveau, C. (2002). Enquêter sur les pratiques sportives des français : tendances lourdes et problèmes méthodologiques. In P. Mignon et G. Truchot (Eds.), Les pratiques sportives en France : enquête 2000 (pp. 135-154). Paris : Ministère des sports, INSEP.

Pujade-Renaud, C. (1983). Le corps de l'enseignant dans la classe. Paris : L'Harmattan.

Rǿnholt, H. (2002). "It's only the sissies..." : analysis of teaching and learning process in physical education : a contribution to the hidden curriculum. Sport, education and society, 7(1), 25-36.

Terret, T., Cogérino, G., \& Rogowski, I. (2006). La mixité en EPS : pratiques et représentations. Paris : Editions Revue EP.S.

Vermersch, P. (1994). L'entretien d'explicitation en formation initiale et en formation continue. Paris : ESF. 
Vermersch, P. (2002). La prise en compte de la dynamique attentionnelle : éléments théoriques.

Expliciter, 43, 27- 39.

Vermersch, P. (2004). Prendre en compte la phénoménalité : propositions pour une psycho phénoménologie. Expliciter, 57, 35-46.

Vigneron, C. (2004). La construction des inégalités de réussite en EPS au Baccalauréat entre filles et garçons. Thèse de doctorat en sciences de l'éducation, Dijon, Université de Bourgogne.

Vigneron, C. (2006). Les inégalités de réussite en EPS entre filles et garçons : déterminisme biologique ou fabrication scolaire? Revue Française de Pédagogie, 154, 111-124.

Wright, J. (1996). The construction of complementarity in physical education. Gender and education, 8(1), 61-69.

\section{NOTES}

1. Direction de l'Évaluation, de la Prospective et de la Performance.

2. Par exemple l'enseignant(e) propose que le but des filles compte double.

\section{RÉSUMÉS}

Les recherches en Éducation Physique et Sportive (EPS) montrent des enseignant-e-s très majoritairement favorables à la mixité, mais elles mettent à jour des mécanismes discriminatoires laissant supposer que ces dernier(e)s seraient sans conscience des inégalités qu'ils/elles provoquent. Le choix d'une étude s'appuyant sur la psychophénoménologie permet de documenter les logiques intrinsèques à l'origine des discriminations: 24 entretiens d'explicitation ont été réalisés auprès d'enseignant-e-s d'EPS afin de leur faire décrire leurs expériences vécues de cours mixtes. Nos résultats montrent que la mixité est toujours subjectivement située : sa forme, choisie par l'enseignant-e, implique en retour des décisions de justice ainsi qu'une vigilance plus ou moins forte du point de vue de l'équité sexuée. Deux études de cas permettront d'illustrer les facteurs subjectifs qui jouent sur cette vigilance.

Researches show that physical education (PE) teachers are overwhelmingly in favor of coeducation. But they update discriminatory mechanisms suggesting that they are unaware of the inequities they cause. A study based on psychophenomenology allows to document the intrinsic logics behind discrimination: 24 explanatory interviews were carried out with PE teachers to let them describe their lived experiences in coeducational classes. Our results show that coeducation is always subjectively located: its form, chosen by the teacher, calls in return for justice decisions and for a more or less strong vigilance concerning gender equity. Two case studies illustrate the subjective factors that influence alertness toward the gender equity. 
INDEX

Mots-clés : égalité filles/garçons, équité, mixité, vigilance

Keywords : awareness, coeducation, equity, gender equality

\section{AUTEURS}

\section{CATHERINE PATINET-BIENAIMÉ}

Centre de Recherche sur l'Innovation en Sport (CRIS, EA 647) - Université Claude Bernard Lyon.

\section{GENEVIÈVE COGERINO}

Centre de Recherche sur l'Innovation en Sport (CRIS, EA 647) - Université Claude Bernard Lyon. 